\title{
Mitochondrial aldehyde dehydrogenase-2 (ALDH2) Glu504Lys polymorphism contributes to the variation in efficacy of sublingual nitroglycerin
}

\author{
Yifeng Li,1,2 Dandan Zhang, ${ }^{1}$ Wei Jin,,2,3 Chunhong Shao, ${ }^{4}$ Pengrong Yan, ${ }^{1}$ \\ Congjian Xu, ${ }^{5}$ Haihui Sheng, ${ }^{2}$ Yan Liu, ${ }^{2,3}$ Jinde $Y u,{ }^{3}$ Yuying Xie, ${ }^{1}$ Yingnan Zhao, ${ }^{1}$ \\ Daru Lu, ${ }^{1}$ Daniel W. Nebert, ${ }^{6}$ Donald C. Harrison, ${ }^{6}$ Wei Huang, ${ }^{2,3}$ and Li Jin',6
}

1State Key Laboratory of Genetic Engineering, School of Life Sciences and Morgan-Tan International Center for Life Sciences, Fudan University, Shanghai, People's Republic of China. ${ }^{2}$ Chinese National Human Genome Center at Shanghai, Shanghai, People's Republic of China ${ }^{3}$ Ruijin Hospital, Shanghai Second Medical University, Shanghai, People's Republic of China. ${ }^{4}$ HuaShan Hospital, Medical Center of Fudan University, Shanghai, People's Republic of China. ${ }^{5}$ Obstetrics and Gynecology Hospital, Medical Center of Fudan University, Shanghai, People's Republic of China. ${ }^{6}$ University of Cincinnati College of Medicine, Cincinnati, Ohio, USA.

\begin{abstract}
Glyceryl trinitrate (GTN), also known as nitroglycerin, has been used to treat angina and heart failure for more than 130 years. Recently, it was shown that mitochondrial aldehyde dehydrogenase-2 (ALDH2) is responsible for formation of NO, the metabolite needed for GTN efficacy. In the present study, we show that the common G-to-A polymorphism in exon 12 of $A L D H 2$ - resulting in a Glu504Lys replacement that virtually eliminates ALDH2 activity in both heterozygotes and homozygotes - is associated with a lack of efficacy of sublingual GTN in Chinese subjects. We also show that the catalytic efficiency $\left(V_{\max } / K_{m}\right)$ of GTN metabolism of the Glu504 protein is approximately 10-fold higher than that of the Lys504 enzyme. We conclude that the presence of the Lys504 allele contributes in large part to the lack of an efficacious clinical response to nitroglycerin; we recommend that this genetic factor be considered when administering nitroglycerin to patients, especially Asians, $30-50 \%$ of whom possess the inactive $A L D H 2 * 2$ mutant allele.
\end{abstract}

\section{Introduction}

Ever since its first clinical application in 1876, glyceryl trinitrate (GTN), also known as nitroglycerin, has been widely used for the management of coronary heart disease. Sublingual GTN administration is the standard treatment for acute angina pectoris, a symptom that is usually relieved within 5 to 10 minutes (1). The physiological mechanism of the therapeutic action of GTN has only recently been explained (2); following metabolism, GTN releases pharmacologically active NO or the NO congener $S$-nitrosothiol, which can activate cyclic GMP (cGMP) and consequently relax vascular smooth muscle (1-3). The exact mechanism underlying its biotransformation remained controversial $(4,5)$ until Stamler and coworkers $(2)$ showed that mitochondrial aldehyde dehydrogenase-2 (ALDH2) is the enzyme principally responsible for GTN bioactivation, leading to the release of NO. This has now been confirmed by the fact that inhibition of ALDH2 contributes to GTN tolerance (6). Also, studies with Aldh2-/- mice (7) have recently shown that mitochondrial ALDH2 is necessary and sufficient for vasoactivity derived from therapeutic levels of GTN.

$A L D H 2$ is one of 19 members of the human $A L D H$ gene family of $\mathrm{NAD}(\mathrm{P})^{+}$-dependent enzymes (8). ALDH2 is the principal enzyme involved in acetaldehyde oxidation at the physiological concentrations typically found when a person consumes alcohol.

Nonstandard abbreviations used: ALDH2, aldehyde dehydrogenase-2; cGMP, cyclic GMP; 1,2-GDN, 1,2-glyceryl dinitrate; GTN, glyceryl trinitrate.

Conflict of interest: The authors have declared that no conflict of interest exists. Citation for this article: J. Clin. Invest. 116:506-511 (2006). doi:10.1172/JCI26564.
In addition to the aforementioned dehydrogenase activity, ALDH2 possesses an esterase activity (9) that catalyzes the hydrolysis of nitroglycerin to generate 1,2-glyceryl dinitrate (1,2-GDN) and nitrite $\left(\mathrm{NO}_{2}^{-}\right)$(2). A common polymorphism in exon 12, in which glutamate is replaced by lysine at position 504 (Glu504Lys), is known to essentially eliminate its dehydrogenase activity and therefore its ability to clear acetaldehyde in homozygotes and to decrease by approximately $94 \%$ the enzyme activity in heterozygotes (10-12). Worldwide, the Lys504 allele has the highest prevalence (30-50\%) in Asian populations, including Chinese people (13). ALDH2 is a tetramer, and 1 defective subunit is sufficient to render the entire enzyme inactive; thus, Glu504 homozygotes $(A L D H 2 * 1 / 1)$ exhibit normal enzymatic activity, Glu504/Lys504 heterozygotes $(A L D H 2 * 1 / 2)$ show approximately $6 \%$ of normal activity, and Lys504 homozygotes $(A L D H 2 * 2 / 2)$ show negligible activity toward acetaldehyde (12). The ALDH2*2 allele is thus inherited as an autosomal dominant trait $(11,12,14)$. This mutation has been considered as a protective factor against the development of alcoholism and may explain distinct alcohol drinking habits across various ethnic populations (13).

Site-directed mutagenesis studies have shown that ALDH2 Cys319 is the essential nucleophilic base for both the dehydrogenase and esterase activities (15). Substituting Lys504 for Glu504, the positive charge of lysine not only weakens the interaction between $\mathrm{NAD}^{+}$and the enzyme, but decreases 10 - to 20fold the nucleophilicity of Cys319 (16). Since NAD ${ }^{+}$and $p$-nitrophenol acetate (the common substrate for measuring ALDH2 esterase activity) share the same binding site (17) and since acetaldehyde is a competitive inhibitor of GTN turnover by ALDH2 


\section{Table 1}

Comparison of parameters in the sublingual GTN efficacious and inefficacious groups

\begin{tabular}{|c|c|c|c|c|}
\hline \multicolumn{2}{|l|}{ Parameters } & $\begin{array}{l}\text { Efficacious } \\
\text { group }\end{array}$ & $\begin{array}{l}\text { Inefficacious } \\
\text { group }\end{array}$ & $\begin{array}{c}P \\
\text { value }\end{array}$ \\
\hline \multicolumn{5}{|l|}{ Sex } \\
\hline & Male & 47 (78.3\%) & $13(21.7 \%)$ & \multirow[b]{2}{*}{0.107} \\
\hline \multirow{5}{*}{$\begin{array}{l}\text { Age (means } \pm \mathrm{SD} \\
\text { Disease severity } \\
\text { Genotype }\end{array}$} & Female & $12(60.0 \%)$ & $8(40.0 \%)$ & \\
\hline & & $64.3 \pm 9.8$ & $65.9 \pm 9.0$ & 0.531 \\
\hline & & $35.0 \pm 10$ & $32.3 \pm 11$ & 0.307 \\
\hline & $A L D H 2 * 1 / 1$ & $40(85.1 \%)$ & $7(14.9 \%)$ & \multirow{2}{*}{0.006} \\
\hline & $\begin{array}{c}A L D H 2 * 1 / 2 \\
\text { and }{ }^{*} 2 / 2\end{array}$ & $19(57.6 \%)$ & $14(42.4 \%)$ & \\
\hline
\end{tabular}

ADisease severity was estimated using a previously reported method (40).

(2), it seems likely that this polymorphism not only affects the dehydrogenase activity but would also affect the esterase activity (18). This prompted us to examine the role of the Glu504Lys mutation in GTN metabolic activation in vitro as well as GTN efficacy in a clinical population.

\section{Results}

Clinical studies. The relationship between interindividual variation in the efficacy of sublingual GTN and this $A L D H 2$ polymorphism was investigated in a Chinese population. As described in Methods, from 568 coronary heart disease patients, we identified 80 patients on sublingual GTN who were qualified for further analysis. We then divided these patients into 2 groups: those for whom GTN was efficacious and those for whom GTN was inefficacious (Table 1). We found no significant differences between the 2 groups (Table 1 ) with regard to sex, age, or severity of the disease. Out of 80 patients, $59(74 \%)$ subjects responded to the medication. Among them, the ALDH2*1/1 homozygotes showed a higher efficacious rate (40 out of 47 , or $85.1 \%$ ) than those carrying at least one $A L D H 2 * 2$ allele (19 out of 33 , or $57.6 \%$ ), resulting in an odds ratio of 4.21 (95\% confidence interval, 1.51-11.7). By $\chi^{2}$ analysis, the efficacy of sublingual GTN was shown to be significantly associated with the $A L D H 2 * 1 / 1$ homozygotes, with $P=0.006$ (2-tailed Pearson's $\chi^{2}$ test) and $P=0.009$ (2-tailed Fisher's exact test) using SPSS version 11.0. A significant association also remained after adjustment for sex, age, and severity of disease. In addition, we resequenced the ALDH2 gene in 30 unrelated healthy Chinese individuals (not shown), and no nonsynonymous mutations in the coding region (including the mitochondrial leader peptide) or splice/junction mutations were found, other than the Glu504Lys. These data indicate that the involvement of other $A L D H 2$ variants that might contribute to variation in sublingual GTN efficacy is unlikely.

The GTN-nonresponsive group comprised 21 (26\%) patients; this incidence of sublingual GTN nonresponders is higher than the incidence of $12 \%$ nonresponders that had been reported in a United States population (19). Considering possible differences in ethnicity and the criteria used for determining sublingual GTN efficacy in these 2 populations, however, we believe that this difference of $26 \%$ versus $12 \%$ is not likely to be significant.

Enzyme kinetics. We next investigated the effect of the Glu504Lys mutation on the kinetic properties of the ALDH2 enzyme in vitro. It is close to impossible to obtain sufficient quantities of the enzyme from blood vessels wherein GTN biotransformation occurs; on the other hand, liver is a reasonable source for ALDH2, given that both ALDH2 in blood vessels and that of liver exhibit similar catalytic properties in GTN biotransformation (2). We therefore collected the livers of naturally aborted fetuses and obtained the purified enzyme of $A L D H 2 * 1 / 1$ and $A L D H 2 * 1 / 2$ liver (Figure 1A) because it was known that hepatic ALDH2 in the fetus resembles that of the adult with regard to catalytic properties of the different genotypes (20). The ALDH2 genotype of each fetus was determined before the ALDH2 protein was purified. Since no Lys/Lys504 homozygous fetus was obtained, we expressed the Lys504 mutant and the Glu504 native enzyme, using the Bac-toBac Baculovirus Expression System (Figure 1B).

Table 2 shows both the ALDH2 dehydrogenase and the esterase activities (GTN conversion). Figure 2 depicts Lineweaver-Burk plots used for the $K_{m}$ and $V_{\max }$ estimations of all 4 types of the ALDH2 enzyme. The parameters of the ALDH2*1/1 (Glu/Glu504) fetal enzyme are similar to those of the recombinant ALDH $2 * 1 / 1$ enzyme. The ALDH2* $1 / 1$ enzyme - from fetal liver or the recombinant form - displayed a markedly lower $\mathrm{K}_{\mathrm{m}}$ and higher $\mathrm{V}_{\max }$ than either the ALDH2*1/2 (Glu/Lys504) fetal enzyme or the ALDH2*2/2 (Lys/Lys504) recombinant enzyme. For ALDH2 dehydrogenase activity, the ALDH $2 * 2 / 2$ was approximately $2 \%$, and the ALDH2* $1 / 2$ was $13-14 \%$ of the ALDH2* $1 / 1$ activity. For the GTN catalytic efficiencies $\left(\mathrm{V}_{\max } / \mathrm{K}_{\mathrm{m}}\right)$, the ALDH2*2/2 was $6-7 \%$, and the ALDH2* $1 / 2$ was $8-15 \%$ of the ALDH2* $1 / 1$ activity. In all forms of the enzymes assayed, the ratio of 1,2-GDN/1,3-GDN was constant at 8:1. Furthermore, the decreased GTN biotransformation in ALDH2* $1 / 2$ heterozygotes was dominant over the ALDH2*1/1 subjects, a finding similar to that of the ALDH2 dehydrogenase activity $(12,14)$. Although the GTN biotransformation catalytic activity was decreased for the heterozygous and homozygous mutants, a relatively higher activity remained, as compared with their respective dehydrogenase activities. Table 2 also shows that the Lys504 enzyme in heterozygotes leads to an approximately 10fold decrease in GTN catalytic efficiency.

\section{Discussion}

The major findings of the present study provide the first evidence that the common G-to-A polymorphism in exon 12 of ALDH2 - resulting in a Glu504Lys replacement - is associ-
A

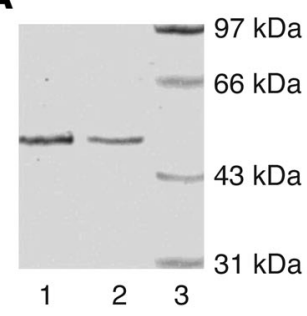

B

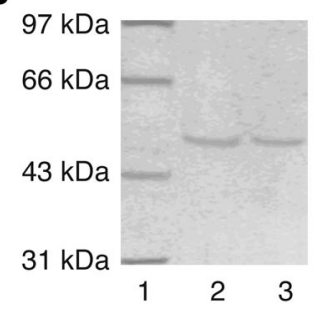

\section{Figure 1}

Purified ALDH2 protein (polyacrylamide gel stained by Coomassie blue). (A) ALDH2 protein purified from human fetal liver. Lane 1, protein from $A L D H 2{ }^{*} 1 / 1$ genotype; lane 2, protein from $A L D H 2{ }^{*} 1 / 2$ genotype; lane 3, molecular weight ladder. (B) Recombinant ALDH2 protein purified via affinity chromatography. Lane 1, molecular weight ladder; lane 2, protein from $A L D H 2{ }^{*} 1 / 1$ genotype; lane 3 : protein from $A L D H 2 * 2 / 2$ genotype. 


\section{Table 2}

ALDH2 kinetic properties as a function of the genotype of fetal liver versus recombinant enzyme

\begin{tabular}{|c|c|c|c|c|}
\hline \multirow[b]{2}{*}{ Genotype encoding enzyme ${ }^{A}$} & \multirow{2}{*}{$\begin{array}{c}\text { Dehydrogenase } \\
\text { activity }^{B} \\
\text { (U/mg protein) }\end{array}$} & \multicolumn{3}{|c|}{ Esterase activity } \\
\hline & & $\begin{array}{c}K_{m} \text { for GTNC } \\
(\mu \mathrm{M})\end{array}$ & $\begin{array}{c}V_{\max } \text { for GTNC } \\
(\mathrm{nmol} / \mathrm{min} / \mathrm{mg})\end{array}$ & $\mathbf{V}_{\max } / K_{m}$ \\
\hline$A L D H 2 * 1 / 1$ (fetal liver) & $0.586 \pm 0.082$ & 8.20 & 5.14 & 0.627 \\
\hline$A L D H 2 * 1 / 2$ (fetal liver) & $0.075 \pm$ & 20.2 & 1.12 & 0.055 \\
\hline ALDH2*1/1 (recombinant) & $0.544 \pm 0.071$ & 11.3 & 4.18 & 0.369 \\
\hline$A L D H 2{ }^{\star} 2 / 2$ (recombinant) & $0.012 \pm 0.002$ & 25.5 & 1.08 & 0.042 \\
\hline
\end{tabular}

${ }^{\mathrm{A}} A L D H 2{ }^{*} 1$ allele encodes Glu504 enzyme. ALDH2*2 allele encodes Lys504 enzyme. ${ }^{\mathrm{B}}$ One unit of ALDH2 activity is defined as the production of $1 \mu \mathrm{mol} \mathrm{NADH} / \mathrm{min}$, in the presence of propionaldehyde as substrate. ${ }^{C}$ The formation of 1,2-GDN and 1,3-GDN from $\left[2-{ }^{-14} \mathrm{C}\right] \mathrm{GTN}$ (assay of GTN metabolic activation) was determined by thin-layer chromatography followed by liquid-scintillation spectrometry $(2,39)$.

ated with a lack of efficacy of sublingual GTN in Chinese subjects. We showed that the catalytic efficiency $\left(V_{\max } / K_{m}\right)$ of GTN metabolism of the Glu504 protein is 10 -fold higher than that of the Lys504 enzyme. We therefore conclude that the presence of the Lys504 allele contributes in part to the failure of the efficacious response to nitroglycerin.

Nitroglycerin has continued to be one of the most commonly used organic nitrates for the management of coronary heart disease. Although chronic GTN therapy leads to endothelial dysfunction $(21,22)$ and GTN does not provide survival benefits, patients who have mastered the proper use of GTN regard it as a "wonder drug" (23). Recently Stamler and coworkers showed that ALDH2 is primarily responsible for the bioactivation of GTN prior to the release of NO $(2,7)$. They showed that vasodilation by GTN, which is dependent on cGMP, can be blocked by ALDH2 inhibitor in vivo and in vitro, whereas the relaxation independent of cGMP was not affected (2). These results were confirmed in studies by Kollau et al. (24) and Zhang et al. (25), indicating that ALDH2 does indeed contribute to, albeit does not exclusively mediate, the observed bioactivation of GTN in mitochondria. Also, the central role of ALDH2 in the biotransformation has been confirmed by the fact that the inhibition of ALDH2 contributes to GTN tolerance (6).

That we did not find 47 out of $47 A L D H 2 * 1 / 1$ homozygotes exhibiting GTN efficacy and 33 out of 33 subjects carrying at least one $A L D H 2 * 2$ allele showing no GTN efficacy indicates that other modifier genes and/or environmental factors must also be reflected in this trait (26). These data also are in agreement with previous reports that other pathways appear to be involved in the metabolism of GTN, such as glutathione $S$-transferase (4) and the 1 or more cytochromes P450 (5). This may help to explain the efficacy of GTN in some patients despite their genetic ALDH2 deficiency. According to the results of functional studies as well as previous reports, the catalytic efficiency of $\mathrm{ALDH} 2$ is much higher than that of these other enzymes, which have been shown to perform the same function during GTN biotransformation (27). From the standpoint of NO biology, mitochondrial GTN reductase activity is significantly greater than mitochondrial NO synthase activity $(28,29)$.

There are several factors involved in the decreased ability of nitroglycerin biotransformation in the $A L D H 2 * 2$ heterozygote. Primarily, the $\mathrm{K}_{\mathrm{m}}$ value increases and the $\mathrm{V}_{\max }$ value decreases drastically in the heterozygote and homozygote mutant ALDH2, as compared with that of the native enzyme. Catalytic efficiency $\left(\mathrm{V}_{\max } / \mathrm{K}_{\mathrm{m}}\right)$ of GTN metabolism of the Glu504 protein is approximately 10 -fold higher than that of the Lys504 enzyme. We believe that the change in kinetic properties is primarily responsible for the decreased GTN biotransformation. Secondly, Xiao et al. reported that the $A L D H 2 * 2$ allele exerts its dominant effect not only by diminishing catalytic activity, but also by increasing its turnover, with the half-life decreased to nearly one-half that of the native enzyme (22 hours versus 14 hours), and this difference in half-life between the native and mutant enzyme can lead to a $34 \%$ decrease in total ALDH2 protein (30).

The functional ALDH2 enzyme requires a tetramer to be active (10); if any of the 4 subunits is the defective Lys504 isotype, the activity of the entire tetramer will be severely compromised $(12,31)$.

Esterase and dehydrogenase activity share the same mechanism - except that the hydride transfer step is not involved in the esterase reaction. Site-directed mutagenesis studies have shown that Cys319 is the essential nucleophile base for both dehydrogenase and esterase activity, with Glu285 as the specific base required to activate Cys319 $(15,32)$. The molecular mechanism underlying the differences in kinetic properties toward aldehyde between the native and mutant enzymes has been intensively studied. The dramatic loss in activity of the Lys504 enzyme was shown to reflect the weakened interaction between $\mathrm{NAD}^{+}$and the enzyme (the dissociation constant of the Lys504 enzyme for $\mathrm{NAD}^{+}$is 50 times larger than that of the native Glu504 form); when Glu504 was replaced by a Gln residue in the human recombinant enzyme, kinetic properties similar to those of the native form were found (16). On the other hand, the lowered nucleophilicity of Cys319 ( 10- to 20-fold decrease), interfered with by the positive charge of Lys504, can be another factor in decreasing enzyme activity (16). The lowered nucleophilicity of Cys319 appears to be the cause of the decreased $V_{\max }$ value of GTN biotransformation by the Lys504 enzyme. Furthermore, it has been reported that the salt bond between Glu504 and Arg492 is necessary for the maintenance of the enzyme's active structure $(33,34)$, and the loss of this salt bond in the Lys504 enzyme would be an additional factor responsible for the decreased half-life of the mutant enzyme.

The previous amino acid nomenclature for ALDH2 has been updated. The well-known Glu487Lys mutation in the ALDH2 protein is actually Glu504Lys, due to an N-terminus mitochondrial leader peptide of 17 amino acids (http://www.ensembl.org/ index.html; http://snpper.chip.org/), which had not previously been accounted for in the numbering of the enzyme's residues. Consequently, all amino acids of this enzyme referred to needed to be corrected by the addition of 17 residues. This is what we have done in the present publication.

Considering the wide use of GTN for the treatment of coronary heart disease, we recommend that this genetic factor be considered when administering nitroglycerin to patients, especially to Asians, $30-50 \%$ of whom possess the inactive $A L D H 2 * 2$ allele. In addition to a DNA test, it is well known that the "flushing 

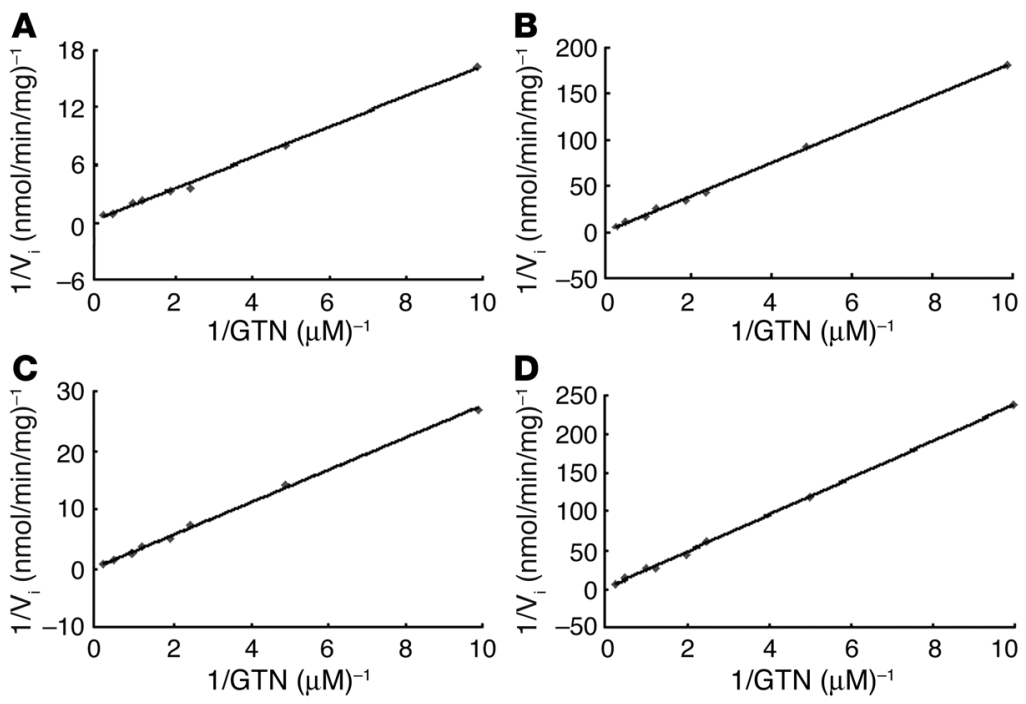

Figure 2

Representative Lineweaver-Burk plots for GTN as the variable substrate for the fetal ALDH2*1/1 (A) and ALDH2* $1 / 2$ (B) and the recombinant ALDH2* $1 / 1$ (C) and ALDH2*2/2 (D) enzymes.

response" to alcohol is an indicator of the ALDH2*2 allele (35). Thus, this test may be a convenient and conservative alternative for the practicing physician in predicting sublingual GTN efficacy if the DNA test is not readily available.

\section{Methods}

Subjects. All subjects were of Han Chinese ethnicity. The protocols for this study were approved by the Ethics Committee of the Chinese National Human Genome Center at Shanghai (Internal Review Board no. 0012), and informed consent was obtained from each subject. A total of 568 patients were identified by coronary arteriography as having coronary heart disease. The following inclusion criteria were then used: (a) stable angina pectoris (according to the diagnostic criteria of WHO); (b) no other diagnosed disease that might show manifestations similar to those of coronary heart disease; (c) sublingual GTN self-administered during acute angina attacks; and (d) no other antiangina drug taken together with GTN. Applying these inclusion criteria, 111 subjects were selected for further analysis. These subjects were divided into 2 groups, those for whom GTN was efficacious and those for whom it was inefficacious, according to a previous report (19), with minor modifications. The response to sublingual GTN was determined using a pain judgment scale of 11 grades -0 indicating no pain and 10 representing maximal pain (a provocative test is less subjective but was not accepted by the recruited subjects because of its lack of safety). The GTNefficacious group met the following criteria: (a) pain level decreasing by at least 2 grades; (b) pain relief occurring within 10 minutes after taking GTN; and (c) length of time of angina being shortened. The GTN-inefficacious group met the following criteria: (a) no remission of pain or decrease of only 1 grade and (b) no change in length of time of angina after sublingual GTN administration (19). Patients who showed a significant reduction of pain only after more than 10 minutes following sublingual GTN administration were excluded because of the well-known rapid clinical effect of this drug (36). This exclusion further decreased our sample size to 80 subjects.

Genotyping. Genomic DNA was extracted from leukocytes, and the DNA sequence spanning the $A L D H 2$ polymorphism was amplified by PCR. Genotyping was performed by direct sequencing, using an ABI 3100 Sequencer (Applied Biosystems).
ALDH2 purification. Overall, 20 naturally aborted fetuses were collected. The $A L D H 2$ genotype of each sample was determined prior to enzyme purification. The purification procedure was conducted following methods reported previously $(17,37)$, with minor modifications. Fetal liver $(50 \mathrm{~g})$ of each genotype was washed in cold $0.9 \% \mathrm{NaCl}$ solution. After homogenization in extraction buffer A $(30 \mathrm{mM}$ potassium phosphate, $1 \mathrm{mM}$ EDTA, $1 \mathrm{mM}$ dithiothreitol, $2 \mathrm{mM}$ benzamidine, and $70 \mathrm{mM}$ 2-morpholinoethane sulfonic acid; $\mathrm{pH}$ 6.0), the mitochondrial fraction was isolated from the homogenate, following instructions in the mitochondria isolation kit (Sigma-Aldrich). The final purified mitochondrial pellet was resuspended and disrupted by sonication. Total mitochondrial protein was loaded onto a DEAE-Sepharose 4B column to remove contaminating proteins. The flow-through fractions with ALDH2 enzyme activity were pooled and loaded onto an $\alpha$-hydroxycyanocinnamate-Sepharose 6B column that had been equilibrated with buffer $\mathrm{A}$. The column was washed until the effluent was free of material absorbing at $280 \mathrm{~nm}$. The column was then washed with a linear $\mathrm{pH}$ gradient ( $\mathrm{pH} 6.0$ to $\mathrm{pH} 7.6)$ by mixing in the chamber buffer $\mathrm{A}$ and buffer $\mathrm{B}(0.42 \mathrm{M}$ potassium phosphate, $1 \mathrm{mM}$ EDTA, $1 \mathrm{mM}$ DTT, $2 \mathrm{mM}$ benzamidine, and $70 \mathrm{mM}$ MES; $\mathrm{pH}$ 7.6). The column was reequilibrated with buffer A. The ALDH2 enzyme activity was specifically eluted by $2 \mathrm{mM}$ $\alpha$-hydroxycyanocinnamic acid in buffer $\mathrm{A}$. The fraction containing ALDH2 activity was concentrated to a final volume of $5.0 \mathrm{ml}$ by ultrafiltration and then dialyzed overnight at $4{ }^{\circ} \mathrm{C}$ against buffer $\mathrm{A}$. The purity of protein was determined by SDS-PAGE, followed by protein staining with Coomassie blue; the purified enzyme was confirmed by protein sequencing. All steps were performed at $4^{\circ} \mathrm{C}$. The enzyme was stored at $-70^{\circ} \mathrm{C}$ in the presence of $20 \%(\mathrm{v} / \mathrm{v})$ glycerol.

$A L D H 2$ expression. From a human liver cDNA library constructed in $\lambda$ gt11 (Clontech), a full-length ALDH2 cDNA was isolated by a 2-step nested-PCR amplification, using 2 sets of primers. For the long-PCR primers, the sense primer was 5 '-AGCTGCGCCCCCATCCCGAGGAAT- $3^{\prime}$, and the antisense primer was $5^{\prime}$-TCGAGTTATAGGAAGCGTTTCAGAG-3'. For the nested-PCR primers, the sense primer was 5'-CACTCGAGGCCCGCTGCGATGTTGC-3', which includes an XhoI restriction site, and the antisense primer was $5^{\prime}$-TCAAGCTTTTATGAGTTCTTCTGAGGCACTTTGAC-3', which includes a HindIII restriction site. The nested-PCR product was digested with XhoI and HindIII and inserted into XhoI/HindIII sites of the pFastbac1 plasmid to form pFastbac1-ALDH2*1 (Figure 3). The recombinant plasmid was identified by both restriction endonuclease digestion and sequencing. The mutation was introduced into the recombinant plasmid by site-directed

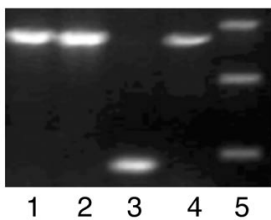

$2500 \mathrm{bp}$

$1000 \mathrm{bp}$

$250 \mathrm{bp}$

\section{Figure 3}

Plasmids containing the inserted ALDH2 cDNA fragment screened by bacterial PCR with universal primer. Lanes 1, 2, and 4, PCR products amplified from bacterial colonies having the recombinant plasmids; lane 3, negative control; lane 5, markers. 
mutagenesis (Mutagene Kit; Takara Bio Inc.) following the manufacturer's instructions, and the mutation was verified by DNA sequencing. Transposition and transfection of the recombinant virus to sf9 cells was carried out according to instructions in the manual of Bac-to-Bac Baculovirus Expression Systems (Invitrogen Corp.). The successful expression of recombinant ALDH2 protein was verified by immunoblotting. For the recombinant protein, we followed the methods of purification and storage, as described above for the human fetal liver ALDH2, but without the isolation of mitochondria. The final enzyme preparation showed a single protein band approximately $54 \mathrm{kDa}$ on $12 \%$ polyacrylamide gel stained by Coomassie Blue A. The enzyme was stored at $-70^{\circ} \mathrm{C}$ in the presence of $20 \%(\mathrm{v} / \mathrm{v})$ glycerol.

Assay of enzyme activities. ALDH2 dehydrogenase activity was assayed at $25^{\circ} \mathrm{C}$ in a Unikon 941 dual-beam spectrophotometer by monitoring $\mathrm{NADH}$ formation from NAD at $340 \mathrm{~nm}$ spectrofluorometrically, as described (38). The assay mixture contained $100 \mathrm{mM}$ Tris-HCL ( $\mathrm{pH}$ 8.5), $1 \mathrm{mM} \mathrm{NAD}$, and $1 \mathrm{mM} 4$-methylpyrazole, and the reaction was started by addition of $1 \mathrm{mM}$ propionaldehyde. One unit of ALDH2 activity is defined as the production of $1 \mu \mathrm{mol} \mathrm{NADH} / \mathrm{min}$. The formation of $1,2-\mathrm{GDN}$ and 1,3-GDN from $\left[2-{ }^{14} \mathrm{C}\right] \mathrm{GTN}$ (assay of GTN conversion) was determined by thin-layer chromatography and liquid-scintillation spectrometry, as reported previously $(2,39)$. Protein concentrations were determined by the Bradford method, with bovine serum albumin as the standard.

Enzyme kinetics. $\mathrm{K}_{\mathrm{m}}$ values were calculated over a range of 8 concentrations of substrate (from $0.1 \mu \mathrm{M}$ to $4 \mu \mathrm{M}$ ), using Lineweaver-Burk plot analysis (Figure 2) derived from the determination of initial velocities. Each assay point was performed at least in triplicate and averaged. SD were less than $15 \%$ of the means.

Statistics. The association analyses (2-tailed Student's $t$ test and $\chi^{2}$ test) were performed using SPSS version 11.0 (SPSS Inc.). The continuous variables were expressed as the means \pm SD and compared with Student's independent $t$ test. The categorical variables were expressed as percentages, and the $\chi^{2}$ test was applied for the determination of significance of an association. The effect of the covariants was examined by multiple logistic regression. Kinetic parameters such as $\mathrm{K}_{\mathrm{m}}$ and $\mathrm{V}_{\max }$ were determined, and catalytic efficiency was expressed as the $V_{\max } / K_{m}$ ratio.

\section{Acknowledgments}

We thank all the human subjects for making this work possible. This work was supported by grants from the Chinese High-Tech Program (863) (2002BA711A10), the Shanghai Science and Technology Committee (03DJ14008, 04DZ14003), the Basic Research Program of China (973) (2004CB518605), the Chinese Ministry of Education (2003), and the NIH (P30 ES06096 to D.W. Nebert and L. Jin).

Received for publication August 11, 2005, and accepted in revised form November 15, 2005.

Address correspondence to: Li Jin, State Key Laboratory of Genetic Engineering, School of Life Sciences and Morgan-Tan International Center for Life Sciences, Fudan University, Shanghai 200433, People's Republic of China. Phone: 86-21-65642800; Fax: 86-2155664388; E-mail: 1jin007@gmail.com. Or: Center for Genome Information, Department of Environmental Health, University of Cincinnati College of Medicine, 3223 Eden Avenue, PO Box 670056, Cincinnati, Ohio 45267-0056, USA. Phone: (513) 5582106; Fax: (513) 558-0071. Or to: Wei Huang, Chinese National Human Genome Center at Shanghai, 250 Bi Bo Road, Shanghai 201203, People's Republic of China. Phone: 86-21-50801795; Fax: 86-21-50801795; E-mail: huangwei@chgc.sh.cn.

Yifeng Li, Dandan Zhang, and Wei Jin contributed equally to this work.
1. Lutz, J.F., and Hurst, J.W. 1998. Hurst's the heart. 9th edition. McGraw-Hill. New York, New York, USA. 260 pp.

2. Chen, Z., Zhang, J., and Stamler, J.S. 2002. Identification of the enzymatic mechanism of GTN bioactivation. Proc. Natl. Acad. Sci. U. S. A. 99:8306-8311.

3. Willerson, J.T., and Cohn, J.N. 2000. Cardiovascular Medicine. 2nd edition. Churchill Livingstone. New York, New York, USA. 2344 pp.

4. Lau, D.T., Chan, E.K., and Benet, L.Z. 1992. Glutathione $S$-transferase-mediated metabolism of glyceryl trinitrate in subcellular fractions of bovine coronary arteries. Pharm. Res. 9:1460-1464.

5. McDonald, B.J., and Bennett, B.M. 1993. Biotransformation of glyceryl trinitrate by rat aortic cytochrome P450. Biochem. Pharmacol. 45:268-270.

6. Sydow, K., et al. 2004. Central role of mitochondrial aldehyde dehydrogenase and reactive oxygen species in GTN tolerance and cross-tolerance. J. Clin. Invest. 113:482-489. doi:10.1172/JCI200419267.

7. Chen, Z., et al. 2005. An essential role for mitochondrial aldehyde dehydrogenase in nitroglycerin bioactivation. Proc. Natl. Acad. Sci. U. S. A. 102:12159-12164.

8. Vasiliou, V., and Nebert, D.W. 2005. Analysis and update of the human aldehyde dehydrogenase (ALDH2) gene family. Hum. Genomics. 2:138-143.

9. Feldman, R.I., and Weiner, H. 1972. Horse liver aldehyde dehydrogenase. II. Kinetics and mechanistic implications of the dehydrogenase and esterase activity. J. Biol. Chem. 247:267-272.

10. Yoshida, A.R., Huang, I.Y., and Ikawa, M. 1984. Molecular abnormality of an inactive aldehyde dehydrogenase variant commonly found in Orientals. Proc. Natl. Acad. Sci. U. S. A. 81:258-261.
11. Crabb, D.W., Edenberg, H.J., Bosron, W.F., and Li, T.K. 1989. Genotypes for aldehyde dehydrogenase deficiency and alcohol sensitivity. The inactive $A L D H 2 * 2$ allele is dominant. J. Clin. Invest. 83:314-316.

12. Nebert, D.W., and Jorge-Nebert, L.F. 2002. Pharmacogenetics and pharmacogenomics. In Emery and Rimoin's Principles and Practice of Medical Genetics. D.L. Rimoin, J.M. Connor, R.E. Pyeritz, and B.R. Korf, editors. Churchill Livingstone. New York, New York, USA. 590-631.

13. Goedde, H.W., et al. 1983. Population genetic studies of aldehyde dehydrogenase isozyme deficiency and alcohol sensitivity. Am. J. Hum. Genet. 35:769-772.

14. Xiao, Q., Weiner, H., Johnston, T., and Crabb, D.W. 1995. The aldehyde dehydrogenase $A L D H 2 * 2$ allele exhibits dominance over $A L D H 2 * 1$ in transduced HeLa cells. J. Clin. Invest. 96:2180-2186.

15. Farrés, J., Wang, T.T., Cunningham, S.J., and Weiner, H. 1995. Investigation of the active-site cysteine residue of rat liver mitochondrial aldehyde dehydrogenase by site-directed mutagenesis. Biochemistry. 34:2592-2598.

16. Farrés, J., et al. 1994. Effects of changing Glu-487 to Lys-487 in rat and human liver mitochondrial aldehyde dehydrogenase. A model to study human (Oriental type) class 2 aldehyde dehydrogenase. J. Biol. Chem. 269:13854-13860.

17. Klyosov, A.A., Rashkovetsky, L.G., Tahir, M.K., and Keung, W.M. 1996. Possible role of liver cytosolic and mitochondrial aldehyde dehydrogenases in acetaldehyde metabolism. Biochemistry. 35:4445-4456.

18. Katalin, F.B., and Regina, P. 1984. Human aldehyde dehydrogenase: catalytic activity in Oriental liver.
Biochem. Biophys. Res. Commun. 118:97-102.

19. Shry, E.A., et al. 2002. Usefulness of the response to sublingual GTN as a predictor of ischemic chest pain in the emergency department. Am. J. Cardiol. 90:1264-1266.

20. Yoshida, A., Shibuya, A., Dave, V., Nakayama, M., and Hayashi, A. 1990. Developmental changes of aldehyde dehydrogenase isozymes in human livers: mitochondrial ALDH2 isozyme is expressed in fetal livers. Experientia. 46:747-750.

21. Gori, T., Mak, S.S., Kelly, S., and Parker, J.D. 2001. Evidence supporting abnormalities in nitric oxide synthase function induced by GTN in humans. J. Am. Coll. Cardiol. 38:1096-1101.

22. Gori, T., et al. 2001. Folic acid prevents GTNinduced nitric oxide synthase dysfunction and nitrate tolerance: a human in vivo study. Circulation. 104:1119-1123.

23. Graboys, T.B., and Lown, B. 2003. Cardiology patient page. GTN: the "mini" wonder drug. Circulation. 108:e78-e79.

24. Kollau, A., et al. 2005. Contribution of aldehyde dehydrogenase to mitochondrial bioactivation of nitroglycerin: evidence for the activation of purified soluble guanylate cyclase through direct formation of nitric oxide. Biochem. J. 385:769-777.

25. Zhang, J., Chen, Z., Cobb, F.R., and Stamler, J.S. 2004. Role of mitochondrial aldehyde dehydrogenase in nitroglycerin-induced vasodilation of coronary and systemic vessels: an intact canine model. Circulation. 110:750-755.

26. Nebert, D.W., and Vesell, E.S. 2004. Advances in pharmacogenomics and individualized drug therapy: exciting challenges that lie ahead. Eur. J. Pharmacol. 500:267-280. 
27. Kozlov, A.V., Dietrich, B., and Nohl, H. 2003. Various intracellular compartments cooperate in the release of nitric oxide from glycerol trinitrate in liver. Br. J. Pharmacol. 139:989-997.

28. Kobzik, L., Stringer, B., Balligand, J.L., Reid, M.B., and Stamler, J.S. 1995. Endothelial type nitric oxide synthase in skeletal muscle fibers: mitochondrial relationships. Biochem. Biophys. Res. Commun. 211:375-381.

29. Lacza, Z., et al. 2001. Mitochondrial nitric oxide synthase is constitutively active and is functionally up-regulated in hypoxia. Free Radic. Biol. Med. 31:1609-1615.

30. Xiao, Q., Weiner, H., and Crabb, D.W. 1996. The mutation in the mitochondrial aldehyde dehydrogenase gene ( $A L D H 2)$, responsible for alcoholinduced flushing, increases turnover of the enzyme tetramers in a dominant fashion. J. Clin. Invest. 98:2027-2032.

31. Wang, X.-P., Sheikh, S., Saigal, D., Robinson, L., and Weiner, H. 1996. Heterotetramers of human liver mitochondrial (class 2) aldehyde dehydrogenase expressed in escherichia coli. J. Biol. Chem. 271:31172-31178.

32. Wang, X.-P., and Weiner, H. 1995. Involvement of glutamate-268 in the active site of human liver mitochondrial aldehyde dehydrogenase, as probed by site-directed mutagenesis. Biochemistry. 34:237-243.

33. Steinmetz, C.G., Xie, P., Weiner, H., and Hurley, T.D. 1997. Structure of mitochondrial aldehyde dehydrogenase: the genetic component of ethanol aversion. Structure. 15:701-711.

34. Zhou, J.H., and Weiner, H. 2000. Basis for half-ofthe-site reactivity and the dominance of the K-487 Oriental subunit over the E-487 subunit in heterotetrameric human liver mitochondrial aldehyde dehydrogenase. Biochemistry 39:12019-12024.

35. Wall, T.L., Thomasson H.R., and Ehlers, C.L. 1996. Investigator-observed alcohol-induced flushing, but not self-report of flushing, is a valid predictor of ALDH2 genotype. J. Stud. Alcohol. 57:267-272.

36. Parker, J.O. 1987. Nitrate therapy in stable angina pectoris. N. Engl. J. Med. 316:1635-1642.

37. Rashkovetsky, L.G., Maret, W., and Klyosov, A.A. 1994. Human liver aldehyde dehydrogenases: new method of purification of the major mitochondrial and cytosolic enzymes and re-evaluation of their kinetic properties. Biochim. Biophys. Acta. 1205:301-307.

38. Jeng, J.J., and Weiner, H. 1991. Purification and characterization of catalytically active precursor of rat liver mitochondrial aldehyde dehydrogenase expressed in Escherichia coli. Arch. Biochem. Biophys. 289:214-222.

39. Brien, J.F., et al. 1986. Biotransformation of glyceryl trinitrate occurs concurrently with relaxation of rabbit aorta. J. Pharmacol. Exp. Ther. 237:608-614.

40. Hadorn, D.C., and Holmes, A.C. 1997. The New Zealand priority criteria project. Part 2: Coronary artery bypass graft surgery. Br. Med. J. 314:135-138. 